\title{
A CONVERSE TO THE GRACE-WALSH-SZEGÖ THEOREM
}

\author{
PETTER BRÄNDÉN AND DAVID G. WAGNER
}

\begin{abstract}
We prove that the symmetrizer of a permutation group preserves stability if and only if the group is orbit homogeneous. A consequence is that the hypothesis of permutation invariance in the Grace-Walsh-Szegö Coincidence Theorem cannot be relaxed. In the process we obtain a new characterization of the Grace-like polynomials introduced by D. Ruelle, and prove that the class of such polynomials can be endowed with a natural multiplication.
\end{abstract}

\section{INTRODUCTION AND MAIN RESULT}

The Grace-Walsh-Szegö Coincidence Theorem is undoubtably one of the most useful phenomena governing the location of zeros of multivariate complex polynomials - see [7]. It is also very robust - after a full century of investigation, no significant improvement on it has been made. (Although significant generalizations to other settings have been obtained [6].) The main purpose of this note is to show that, in one sense at least, no such improvement is possible.

A circular region is a proper subset of the complex plane that is bounded by either a circle or a straight line, and is either open or closed. A polynomial is multiaffine provided that each variable occurs at most to the first power.

Proposition 1 (Grace-Walsh-Szegö [5, 9, 10). Let $f \in \mathbb{C}\left[z_{1}, \ldots, z_{n}\right]$ be a multiaffine polynomial that is invariant under all permutations of the variables. Let $\mathcal{A} \subset \mathbb{C}$ be a circular region. Assume that either $\mathcal{A}$ is convex or that the degree of $f$ is n. For any $\zeta_{1}, \ldots, \zeta_{n} \in \mathcal{A}$ there is a $\zeta \in \mathcal{A}$ such that

$$
f\left(\zeta_{1}, \ldots, \zeta_{n}\right)=f(\zeta, \ldots, \zeta) .
$$

The question we address is: for which permutation groups $G \leq \mathfrak{S}_{n}$ does an analogue of the Grace-Walsh-Szegö Theorem hold? That is, can one relax the hypothesis that $f$ is invariant under all permutations of the variables by requiring only that $f$ be invariant under the permutations in a subgroup $G$ ? (As usual, the action of $\mathfrak{S}_{n}$ on $\mathbb{C}\left[z_{1}, \ldots, z_{n}\right]$ is defined for each $\sigma \in \mathfrak{S}_{n}$ by $\sigma\left(z_{i}\right)=z_{\sigma(i)}$ and algebraic extension, and this restricts to define an action of any subgroup.) If $G \leq \mathfrak{S}_{n}$ then a polynomial $f \in \mathbb{C}\left[z_{1}, \ldots, z_{n}\right]$ is $G$-invariant if $\sigma(f)=f$ for all $\sigma \in G$. Let $\mathcal{H}$ denote the open upper half-plane. Our main theorem is the following:

2000 Mathematics Subject Classification. 20B10, 30C15.

Key words and phrases. Grace-Walsh-Szegő coincidence theorem, homogeneous groups, Gracelike polynomials, stable polynomials.

The first author was partially supported by the Göran Gustafsson Foundation and the second author was supported by NSERC Discovery Grant OGP0105392. 
Theorem 2. Let $G \leq \mathfrak{S}_{n}$ be a permutation group. Suppose that for any multiaffine $G$-invariant polynomial $f \in \mathbb{C}\left[z_{1}, \ldots, z_{n}\right]$ and any $\zeta_{1}, \ldots, \zeta_{n} \in \mathcal{H}$ there is a $\zeta \in \mathcal{H}$ such that

$$
f\left(\zeta_{1}, \ldots, \zeta_{n}\right)=f(\zeta, \ldots, \zeta)
$$

Then every $G$-invariant polynomial is also $\mathfrak{S}_{n}$-invariant (i.e., symmetric).

\section{Stability PRESERVERs and Grace-Like polynomials}

In order to prove Theorem 2 we relate the problem at hand to two similar problems, which we now describe.

A multivariate polynomial $f \in \mathbb{C}\left[z_{1}, \ldots, z_{n}\right]$ is stable provided that it is nonvanishing on $\mathcal{H}^{n}$. We say that a linear operator, $T$, on polynomials preserves stability if $T(f)$ is stable or identically zero whenever $f$ is stable. We are interested in whether a linear transformation of the form

$$
T=\sum_{\sigma \in \mathfrak{S}_{n}} c_{\sigma} \sigma
$$

preserves stability, in which $\left\{c_{\sigma}: \sigma \in \mathfrak{S}_{n}\right\}$ are complex numbers. The following proposition gives a point of attack on such questions.

Proposition 3 (Borcea-Brändén [2]). Let $\mathcal{M}_{n}$ be the space of complex multiaffine polynomials in $n$ variables. For a linear transformation $T: \mathcal{M}_{n} \rightarrow \mathcal{M}_{n}$ whose image is at least two-dimensional the following are equivalent:

(1) T preserves stability;

(2) the polynomial $T\left(\prod_{i=1}^{n}\left(z_{i}+w_{i}\right)\right)$ in $\mathcal{M}_{2 n}$ is stable.

(In part (2) of Proposition 3, $T$ acts on $\mathcal{M}_{2 n} \simeq \mathcal{M}_{n} \otimes \mathcal{M}_{n}$ via its given action on the $\mathcal{M}_{n}$ factor supported on the $z$ variables.)

The study of stability-preserving operators of the form (2.1) was initiated by Ruelle [8] from a slightly different point of view. He studied multiaffine complex polynomials $P\left(z_{1}, \ldots, z_{m}, w_{1}, \ldots, w_{n}\right)$ that are nonvanishing whenever $z_{1}, \ldots, z_{m}$ are separated from $w_{1}, \ldots, w_{n}$ by a circle of the Riemann sphere, and termed such polynomials Grace-like. A reformulation of the Grace-Walsh-Szegö Theorem is that the polynomial

$$
\frac{1}{n !} \sum_{\sigma \in \mathfrak{S}_{n}} \prod_{j=1}^{n}\left(z_{\sigma(j)}-w_{j}\right)
$$

is Grace-like. Ruelle [8] proved that if all the variables in a Grace-like polynomial $P$ actually do occur then $m=n$ and

$$
P\left(z_{1}, \ldots, w_{n}\right)=\sum_{\sigma \in \mathfrak{S}_{n}} c_{\sigma} \prod_{j=1}^{n}\left(z_{\sigma(j)}-w_{j}\right)
$$

for some complex numbers $\left\{c_{\sigma}: \sigma \in \mathfrak{S}_{n}\right\}$.

The following proposition relates Grace-like polynomials to stability-preserving linear transformations.

Proposition 4. Let $\left\{c_{\sigma}: \sigma \in \mathfrak{S}_{n}\right\}$ be complex numbers. The following are equivalent: 
(1) the polynomial

$$
P\left(z_{1}, \ldots, z_{n}, w_{1}, \ldots, w_{n}\right)=\sum_{\sigma \in \mathfrak{S}_{n}} c_{\sigma} \prod_{j=1}^{n}\left(z_{\sigma(j)}-w_{j}\right)
$$

is Grace-like;

(2) the linear operator defined by $T=\sum_{\sigma \in \mathfrak{S}_{n}} c_{\sigma} \sigma$ preserves stability;

(3) the polynomial

$$
Q\left(z_{1}, \ldots, z_{n}, w_{1}, \ldots, w_{n}\right)=\sum_{\sigma \in \mathfrak{S}_{n}} c_{\sigma} \prod_{j=1}^{n}\left(z_{\sigma(j)}+w_{j}\right)
$$

is stable.

Proof. The equivalence of (2) and (3) is a special case of Proposition 3.

Clearly (1) implies (3) since the real line separates $z_{1}, \ldots, z_{n}$ from $-w_{1}, \ldots,-w_{n}$ whenever $z_{1}, \ldots, w_{n} \in \mathcal{H}$.

To see that (3) implies (1), assume that $Q$ is stable and that $z_{1}, \ldots, z_{n}$ and $w_{1}, \ldots, w_{n}$ are separated by a circle of the Riemann sphere. By symmetry we may assume that $z_{1}, \ldots, z_{n}$ lie in a convex open circular domain $\mathcal{A}$, and $w_{1}, \ldots, w_{n}$ all lie in the interior of the complement of $\mathcal{A}$. Let

$$
\phi(z)=\frac{a z+b}{c z+d}, \quad a d-b c \neq 0,
$$

be a Möbius transformation that maps $\mathcal{A}$ to $\mathcal{H}$. Then

$$
P\left(z_{1}, \ldots, w_{n}\right)=P\left(\phi\left(z_{1}\right), \ldots, \phi\left(w_{n}\right)\right)(a d-b c)^{n} \prod_{j=1}^{n}\left(c z_{j}+d\right)\left(c w_{j}+d\right)
$$

(see [8, Proposition 5]). However,

$$
\begin{aligned}
& P\left(\phi\left(z_{1}\right), \ldots, \phi\left(z_{n}\right), \phi\left(w_{1}\right), \ldots, \phi\left(w_{n}\right)\right) \\
= & Q\left(\phi\left(z_{1}\right), \ldots, \phi\left(z_{n}\right),-\phi\left(w_{1}\right), \ldots,-\phi\left(w_{n}\right)\right)
\end{aligned}
$$

and $\phi\left(z_{1}\right), \ldots,-\phi\left(w_{n}\right) \in \mathcal{H}$, so that $(3)$ implies that $P\left(z_{1}, \ldots, w_{n}\right) \neq 0$, which proves (1).

A consequence of Proposition 4 is that we may view the class of Grace-like polynomials as a multiplicative sub-semigroup of the group ring $\mathbb{C}\left[\mathfrak{S}_{n}\right]$. Indeed, by Proposition 4 we may identify the class of Grace-like polynomials with the set of elements $\sum_{\sigma \in \mathfrak{S}_{n}} c_{\sigma} \sigma \in \mathbb{C}\left[\mathfrak{S}_{n}\right]$ for which the corresponding linear operator on $\mathcal{M}_{n}$ preserves stability. Since such linear operators are closed under composition the claim follows.

\section{Proof of the main Result}

A permutation group $G \leq \mathfrak{S}_{n}$ is $k$-homogeneous provided that for any two subsets $A, B \subseteq\{1, \ldots, n\}$ with $|A|=|B|=k$ there is a $\sigma \in G$ such that $\{\sigma(a)$ : $a \in A\}=B$. The permutation group $G$ is homogeneous if it is $k$-homogeneous for all $k$. The $G$-symmetrizer of a permutation group $G$ acting on $\{1, \ldots, n\}$ is the linear transformation of the form (2.1) defined by

$$
T_{G}=\frac{1}{|G|} \sum_{\sigma \in G} \sigma .
$$


Note that $T_{G}=T_{\mathfrak{S}_{n}}$ if and only if $G$ is homogeneous.

A homogeneous permutation group is necessarily transitive. More generally, denote the orbits of $G \leq \mathfrak{S}_{n}$ acting on $\{1, \ldots, n\}$ by $S_{1}, S_{2}, \ldots, S_{r}$. Following [3] we say that $G$ is orbit homogeneous provided that for any two subsets $A, B \subseteq$ $\{1, \ldots, n\}$ with $\left|A \cap S_{i}\right|=\left|B \cap S_{i}\right|$ for each $1 \leq i \leq r$ there is a $\sigma \in G$ such that $\{\sigma(a): a \in A\}=B$. If $\mathfrak{S}\left(S_{i}\right)$ denotes the symmetric group on the set $S_{i}$, then the operators $T_{\mathfrak{S}\left(S_{i}\right)}$ commute, and $G$ is orbit homogeneous if and only if $T_{G}=T_{\mathfrak{S}\left(S_{1}\right)} \circ \cdots \circ T_{\mathfrak{S}\left(S_{r}\right)}$.

For our main result we require the following elementary facts.

Lemma 5 (See Section 2 of [4). Assume that $f \in \mathbb{C}\left[z_{1}, \ldots, z_{n}\right]$ is stable. Then

(1) any polynomial obtained from $f$ by fixing some of the variables to values in the closed upper half-plane is stable or identically zero;

(2) any polynomial obtained from $f$ by setting some of the variables equal to one another is stable.

Theorem 6. For a permutation group $G \leq \mathfrak{S}_{n}$ the following are equivalent:

(1) for any multiaffine stable polynomial $f \in \mathcal{M}_{n}, T_{G}(f)$ is stable;

(2) the permutation group $G$ is orbit homogeneous.

Proof. Let the orbits of $G$ acting on $\{1, \ldots, n\}$ be $S_{1}, \ldots, S_{r}$, and let $\left|S_{i}\right|=s_{i}$ for each $1 \leq i \leq r$.

For the easy direction, assume (2). Since $G$ is orbit homogeneous, $T_{G}=T_{\mathfrak{S}\left(S_{1}\right)} \circ$ $\cdots \circ T_{\mathfrak{S}\left(S_{r}\right)}$. That each $T_{\mathfrak{S}\left(S_{i}\right)}$ preserves stability follows from Proposition 4 since the polynomial (2.2) is Grace-like. This establishes (1).

For the converse, assume (1). To prove that $G$ is orbit homogeneous consider any $r$-tuple of natural numbers $\mathbf{a}=\left(a_{1}, \ldots, a_{r}\right)$ such that $a_{i} \leq s_{i}$ for each $1 \leq i \leq r$. A subset $A \subseteq\{1, \ldots, n\}$ such that $\left|A \cap S_{i}\right|=a_{i}$ for each $1 \leq i \leq r$ is said to have profile a. We must show that $G$ has a single orbit in its action on subsets with profile $\mathbf{a}$. We do this by induction on $|\mathbf{a}|=a_{1}+\cdots+a_{r}$. The basis of induction, the case $|\mathbf{a}| \leq 1$, is a restatement of the fact that the orbits of $G$ acting on $\{1, \ldots, n\}$ are $S_{1}, \ldots, S_{r}$.

The induction step falls into two cases

(i) there is a unique index $1 \leq i \leq r$ such that $a_{i}>0$; or

(ii) there are at least two indices $1 \leq i<j \leq r$ such that $a_{i}>0$ and $a_{j}>0$.

For case (i) of the induction step we can re-index the elements of $\{1, \ldots, n\}$ and the orbits of $G$ on points so that $a_{1}=k>0$ and $S_{1}=\{1, \ldots, m\}$. Let $\mathcal{K}$ be any orbit of $G$ acting on $k$-subsets of $S_{1}$. By re-indexing the variables we may assume that $\{1, \ldots, k\} \in \mathcal{K}$. From the hypothesis (1) it follows that

$$
F(\mathbf{z})=T_{G}\left(\left(z_{1}+1\right)\left(z_{2}+1\right) \cdots\left(z_{k}+1\right)\right)
$$

is stable. (The notation $\mathbf{z}$ is short for $\left(z_{1}, \ldots, z_{n}\right)$.) From the induction hypothesis it follows that

$$
F(\mathbf{z})=\frac{1}{|\mathcal{K}|} \sum_{S \in \mathcal{K}} \mathbf{z}^{S}+\sum_{j=0}^{k-1} e_{j}\left(S_{1}\right) \frac{\left(\begin{array}{c}
k \\
j
\end{array}\right)}{\left(\begin{array}{c}
m \\
j
\end{array}\right)},
$$

in which $e_{j}\left(S_{1}\right)=\sum_{J \subseteq S_{1}:|J|=j} \mathbf{z}^{J}$ is the $j$-th elementary symmetric function of the variables $\left\{z_{v}: v \in S_{1}\right\}$ and $\mathbf{z}^{J}=\prod_{j \in J} z_{j}$.

Recall that the Newton inequalities say that if all the zeros of a polynomial $\sum_{j=0}^{d}\left(\begin{array}{l}d \\ j\end{array}\right) b_{j} t^{j}$ are real then $b_{j}^{2} \geq b_{j-1} b_{j+1}$ for all $1 \leq j \leq d-1$. The univariate 
polynomial $p(t)$ obtained from $F$ by setting $z_{1}=\ldots=z_{k}=t$ and $z_{j}=0$ for all $j>k$ is stable by Lemma 5 which means that all its zeros are real. Now

$$
p(t)=\frac{1}{|\mathcal{K}|} t^{k}+\frac{k^{2}}{\left(\begin{array}{c}
m \\
k-1
\end{array}\right)} t^{k-1}+\frac{k^{2}(k-1)^{2}}{4\left(\begin{array}{c}
m \\
k-2
\end{array}\right)} t^{k-2}+\cdots,
$$

so the Newton inequalities for the top three coefficients imply

$$
|\mathcal{K}| \geq \frac{1}{2} \frac{m-k+2}{m-k+1}\left(\begin{array}{c}
m \\
k
\end{array}\right)>\frac{1}{2}\left(\begin{array}{c}
m \\
k
\end{array}\right) .
$$

Thus, every orbit of $G$ acting on $k$-subsets of $S_{1}$ contains strictly more than half of the total number of $k$-subsets of $S_{1}$. Therefore, there is only one such orbit. This completes case (i) of the induction step.

For case (ii) of the induction step, let $A \subseteq\{1, \ldots, n\}$ be such that $\left|A \cap S_{i}\right|=a_{i}$ for each $1 \leq i \leq r$. Re-index the orbits of $G$ acting on points so that $a_{i}>0$ if and only if $1 \leq i \leq p$, where $p \geq 2$ since we are in case (ii). Let $\mathcal{K}$ be the orbit containing $A$ in the action of $G$ on subsets with profile $\mathbf{a}$. By the hypothesis (1) it follows that

$$
F(\mathbf{z})=T_{G}\left(\prod_{v \in A}\left(z_{v}+1\right)\right)
$$

is stable. From the induction hypothesis it follows that

$$
F(\mathbf{z})=\prod_{i=1}^{p} F_{i}\left(S_{i}\right)+\frac{1}{|\mathcal{K}|} \sum_{B \in \mathcal{K}} \mathbf{z}^{B}-\prod_{i=1}^{p} \frac{e_{a_{i}}\left(S_{i}\right)}{\left(\begin{array}{c}
s_{i} \\
a_{i}
\end{array}\right)}
$$

in which each $F_{i}$ is a multiaffine polynomial in the variables indexed by $S_{i}$ - in fact

$$
F_{i}\left(S_{i}\right)=\sum_{j=0}^{a_{i}} \frac{\left(\begin{array}{c}
a_{i} \\
j
\end{array}\right)}{\left(\begin{array}{c}
s_{i} \\
j
\end{array}\right)} e_{j}\left(S_{i}\right) .
$$

Next, obtain $G(\mathbf{z})$ from $F(\mathbf{z})$ by specializing $z_{v}=0$ for all $v \notin A$. The result is

$$
G(\mathbf{z})=\prod_{i=1}^{p} F_{i}\left(S_{i} \cap A\right)+\left(\frac{1}{|\mathcal{K}|}-\prod_{i=1}^{p}\left(\begin{array}{c}
s_{i} \\
a_{i}
\end{array}\right)^{-1}\right) \mathbf{z}^{A} .
$$

By Lemma $5, G(\mathbf{z})$ is stable. Re-index the elements of $\{1, \ldots, n\}$ so that $i \in S_{i} \cap A$ for all $1 \leq i \leq p$, and obtain $H\left(z_{1}, \ldots, z_{p}\right)$ from $G(\mathbf{z})$ by setting $z_{v}=1$ for all $v \in A$ with $p<v$. By Lemma $5, H\left(z_{1}, \ldots, z_{p}\right)$ is stable. From the form of $G(\mathbf{z})$ we see that

$$
H\left(z_{1}, \ldots, z_{p}\right)=\prod_{i=1}^{p}\left(b_{i}+c_{i} z_{i}\right)+C\left(\frac{1}{|\mathcal{K}|}-\prod_{i=1}^{p}\left(\begin{array}{c}
s_{i} \\
a_{i}
\end{array}\right)^{-1}\right) z_{1} \cdots z_{p}
$$

in which the $b_{i}, c_{i}$, and $C$ are strictly positive reals (and $p \geq 2$ is an integer). The polynomial

$$
z_{1} \cdots z_{p} H\left(z_{1}^{-1}, \ldots, z_{p}^{-1}\right)=\prod_{i=1}^{p}\left(b_{i} z_{i}+c_{i}\right)+C\left(\frac{1}{|\mathcal{K}|}-\prod_{i=1}^{p}\left(\begin{array}{c}
s_{i} \\
a_{i}
\end{array}\right)^{-1}\right)
$$

is also stable. Upon specializing the variables so that $z_{i}=b_{i}^{-1}\left(z-c_{i}\right)$, Lemma 5 implies that the resulting polynomial is stable - that is

$$
z^{p}+C\left(\frac{1}{|\mathcal{K}|}-\prod_{i=1}^{p}\left(\begin{array}{c}
s_{i} \\
a_{i}
\end{array}\right)^{-1}\right) \text {. }
$$


If $p \geq 3$ then stability of this polynomial implies that

$$
|\mathcal{K}|=\prod_{i=1}^{p}\left(\begin{array}{c}
s_{i} \\
a_{i}
\end{array}\right) .
$$

If $p=2$ then stability of (3.1) implies that

$$
|\mathcal{K}| \geq \prod_{i=1}^{p}\left(\begin{array}{c}
s_{i} \\
a_{i}
\end{array}\right) .
$$

In either case there is only one orbit of $G$ acting on subsets with profile a. This completes the induction step, and the proof.

Homogeneous permutation groups have been classified, by Chevallay (unpublished) and by Beaumont and Peterson 1. Besides the symmetric and alternating groups there are only four sporadic examples. Direct products of homogeneous groups are orbit homogeneous, but there are others. For example, let $\phi: \mathfrak{S}_{n} \rightarrow \mathfrak{S}(\{n+1, n+2\})$ be the group homomorphism with the alternating group $\mathfrak{A}_{n}$ as kernel. If $n \geq 3$ then the map $\sigma \mapsto \sigma \phi(\sigma)$ identifies $\mathfrak{S}_{n}$ with an orbit homogeneous subgroup of $\mathfrak{S}_{n+2}$. Peter Cameron (personal communication, unpublished) has classified orbit homogeneous groups with two orbits. The general case is perhaps an interesting open problem.

Proof of Theorem [. Suppose that $G$ is as in the statement of Theorem 2, We prove that $G$ is homogeneous, from which the conclusion of Theorem 2 follows.

Suppose that $G$ is not transitive. Then we may partition $\{1, \ldots, n\}$ into two disjoint sets $A, B$ so that the polynomial

$$
\frac{1}{|A|} \sum_{j \in A} z_{j}-\frac{i}{|B|} \sum_{j \in B} z_{j}
$$

is $G$-invariant and constitutes a counterexample to the hypothesis in Theorem 2 Hence $G$ is transitive.

We next prove that the polynomial

$$
F(\mathbf{z}, \mathbf{w})=\frac{1}{|G|} \sum_{\sigma \in G} \prod_{j=1}^{n}\left(z_{\sigma(j)}+w_{j}\right)
$$

is stable. By Proposition 4 and Theorem 6 , this will complete the proof. Suppose that $\mathbf{z}_{0}, \mathbf{w}_{0} \in \mathcal{H}^{n}$ are such that $F\left(\mathbf{z}_{0}, \mathbf{w}_{0}\right)=0$. The polynomial $F\left(\mathbf{z}, \mathbf{w}_{0}\right)$ is $G$ invariant, so by hypothesis there is a $\zeta \in \mathcal{H}$ such that

$$
0=F\left(\zeta, \ldots, \zeta, \mathbf{w}_{0}\right)=\prod_{j=1}^{n}\left(\zeta+w_{j}^{0}\right), \quad \mathbf{w}_{0}=\left(w_{1}^{0}, \ldots, w_{n}^{0}\right),
$$

which is a contradiction since $\zeta+w_{j}^{0} \in \mathcal{H}$ for all $1 \leq j \leq n$.

Acknowledgments. This research stemmed from the authors' participation in the Programme on Combinatorics and Statistical Mechanics at the Isaac Newton Institute, Cambridge, January to June 2008. We thank the staff and administration of the Institute and the other organizers of the Programme for providing a superb environment for research. We also thank Andrea Sportiello for asking the right question, and Peter Cameron for his insights on group theory and the references [1, 3. 


\section{REFERENCES}

[1] R. A. Beaumont, R. P. Peterson, Set-transitive permutation groups, Canad. J. Math. 7 (1955), $35-42$.

[2] J. Borcea, P. Brändén, The Lee-Yang and Pólya-Schur programs. I. Linear operators preserving stability, arXiv:0809.0401

[3] P. J. Cameron, A. W. Dent, Orbit homogeneity in permutation groups, Bull. London Math. Soc. 38 (2006), 587-596.

[4] Y. Choe, J. Oxley, A. Sokal, D. G. Wagner, Homogeneous multivariate polynomials with the half-plane property, Adv. Appl. Math. 32 (2004), 88-187.

[5] J. H. Grace, The zeros of a polynomial, Proc. Cambridge Philos. Soc. 11 (1902), 352-357.

[6] L. Hörmander, On a theorem of Grace, Math. Scand. 2 (1954), 55-64.

[7] Q. I. Rahman, G. Schmeisser, "Analytic Theory of Polynomials", Clarendon Press, Oxford, 2002.

[8] D. Ruelle, Grace-like polynomials, in "Foundations of computational mathematics (Hong Kong, 2000)," pp. 405-421, World Sci. Publ. Co. Inc., River Edge, NJ, 2002.

[9] G. Szegö, Bemerkungen zu einem Satz von J. H. Grace über die Wurzeln algebraischer Gleichungen, Math. Z. 13 (1922), 28-55.

[10] J. L. Walsh, On the location of the roots of certain types of polynomials, Trans. Amer. Math. Soc. 24 (1922), 163-180.

Department of Mathematics, Royal Institute of Technology, SE-100 44 Stockholm, SWEDEN

E-mail address: pbranden@math.kth.se

Department of Combinatorics and Optimization, University of Waterloo, Ontario, CANADA N2L 3G1

E-mail address: dgwagner@math. waterloo.ca 